\title{
FIXAÇÃO DE FÓSFORO POR UM LATOSSOLO E DE'TERMINAÇÃO DO VALOR «X»
}

\author{
Airton Manzano ** \\ Helvécio De Polli ** \\ JEZIEL C. Freire ** \\ LAFAYETTE F. Sobral ** \\ MAURÍcio DE SOUZA** \\ NELSON VENTORIN ** \\ Francisco De A. F. DE Mello ***
}

\section{RESUMO}

Este trabalho se refere a um ensaio conduzido em laboratório para avaliar a capacidade de fixação de fosfato dos horizontes $A_{1}$ $(0-22 \mathrm{~cm}), \mathrm{A}_{3}(22-56 \mathrm{~cm})$ e $\mathrm{B}_{22}(155-200 \mathrm{~cm})$ de Lotossolo Roxo Distrófico. Foi, também, determinado o valor " $\mathrm{X}$ " de WAUGH \& FITTS (1966) dos três horizontes.

Os principais resultados são apresentados a seguir:

1 - $\mathrm{O}$ horizonte $\mathrm{B}_{22}$ foi o que apresentou maior capacidade de fixação de fósforo, seguido pelo $A_{3}$ e, finalmente, pelo $A_{1}$.

2 - Os valores " $X$ " encontrados foram: $350 \mathrm{ppm}, 225 \mathrm{ppm}$ e 175 ppm para os horizontes $B_{22}, A_{3}$ e $A_{1}$, respectivamente.

3 - Houve uma relação muito estreita entre as quantidades de $\mathrm{P}$ adicionadas e as fixadas pelos três horizontes.

\section{INTRODUÇÃO}

De acordo com HEMWALL (1957) o fenômeno da fixação do fósforo pelo solo foi observado, pela primeira vez, na Europa em 1850. Dessa data até o presente muitos trabalhos já foram efetuados a respeito, estabelecendo-se conceitos e interpretações de várias naturezas que ainda não esclareceram devidamente o problema.

* Entregue para publicação em 30.12.1976.

* Alunos do Curso de Pós-Graduação da Escola Superior de Agricultura "Luiz de Queiroz", Piracicaba, 1975.

** Professor de Fertilidade do Solo, da Escola Superior de Agricultura "Luiz de Queiroz". 
Quando fosfatos são adicionados ao solo grande parte é fortemente insolubilizado tornando-se não disponível às plantas por um lapso de tempo variável e desconhecido. Desse modo, o fenômeno se torna um problema importante da química e da físico-química do solo com enorme influência sobre a fertilidade do mesmo.

Neste trabalho são apresentados os resultados obtidos em um estudo de laboratório sobre a fixação de fosfato por três horizontes de um Latossolo Roxo Distrófico, bem como os valres " $\mathrm{X}$ " de WAUGH \& FITTS (1966).

\section{REVISÃO BIBLIOGRÁFICA}

Segundo KARDOS (1964) os tipos de reação através dos quais o fósforo é fixado podem ser colocados em três grupos: adsorção, precipitação e substituição isomorfa.

De acordo com HSU (1965), em solos ácidos, a precipitação ocorre quando o fosfato remove o $\mathrm{Al}^{3+}$ ou o $\mathrm{Fe}^{3+}$ da rede cristalina precipitando-se em uma nova fase. A adsorção é um caso especial no qual o $\mathrm{Al}^{3+}$ e o $\mathrm{Fe}^{3+}$ permanecem como constituintes da fase original e reagem com os ions fasfato através de forças residuais existentes nas superfícies das partículas. A penetração do fósforo na rede cristalina, substituindo o $\mathrm{SiO}_{2}$ ou deslocando hidroxilas, foi verificado por STOUT (1939), na haloisita, e confirmado depois por COLE \& JACKSON (1950).

HEMWALL (1957) diz que os componentes dos solos ácidos responsáveis pela fixação do fósforo são os compostos de ferro e de alumínio e os argilo-minerais. Os compostos fosfatados de ferro e de alumínio compreendem não só os fosfatos insolúveis desses metais mas também os íons fosfato adsorvidos aos óxidos hidratados dos mesmos.

Os dados existentes na literatura sobre a influência da matéria orgânica obre a fixação do fósforo são contraditórios (ver DALTON et al, 1952; BUCKMAN \& BRADY, 1968; LEAL \& VELLOSO, 1973; LOURENÇO, 1973).

No Brasil já existe um número elevado de trabalhos relativos à fixação do fósforo, efetuados por diferentes processos. O método de WAUGH \& FITTS (1966) também já tem sido empregado (MAGALHÃES, 1974; REZENDE, 1974; SOBRAL, 1975) .

\section{MATERIAIS E MÉTODOS}

Foram utilizadas amostras de três horizontes - $A_{1}(0-22 \mathrm{~cm})$, $\mathrm{A}_{3}(22-56 \mathrm{~cm})$ e $\mathrm{B}_{22}(155-200 \mathrm{~cm})$ - de um Latossolo Roxo Distrófico, 
do Município de Laras, Estado de Minas Gerais, cujas características químicas e físicas estão contidas nas Tabelas 1 e 2.

\begin{tabular}{ccccccccc}
\hline & \multicolumn{7}{c}{ e.mg/100ml } & T.F.S.A. \\
\cline { 2 - 6 } & & $\mathrm{Ca}^{2+}+\mathrm{Mg}^{2+}$ & $\mathrm{K}^{+}$ & $\mathrm{Na}^{+}$ & $\mathrm{Al}^{3+}$ & $\mathrm{H}$ & $\mathrm{C} \%$ & $\mathrm{pH}$ \\
\hline $\mathrm{A}_{1}$ & 0,30 & 0,05 & 0,04 & 1,50 & 12,50 & 3,39 & 4,6 \\
$\mathrm{~A}_{3}$ & 0,30 & 0,01 & 0,02 & 0,40 & 5,20 & 1,88 & 4,8 \\
$\mathrm{~B}_{22}$ & 0,20 & 0,01 & 0,02 & 0,10 & 3,10 & 0,85 & 5,0 \\
\hline
\end{tabular}

Tabela 1 - Características químicas dos horizontes $A_{1}, A_{3}$ e $B_{22}$ do solo utilizado.

\begin{tabular}{ccccc}
\hline Horizonte & Areia $\%$ & Silte $\%$ & Argila $\%$ & St, $\mathrm{m}^{2} / \mathrm{g}$ \\
\hline $\mathrm{A}_{1}$ & 15,25 & 2,10 & 82,65 & 53,00 \\
$\mathrm{~A}_{3}$ & 10,60 & 2,25 & 87,15 & 76,00 \\
$\mathrm{~B}_{22}$ & 10,40 & 3,55 & 86,05 & 63,00 \\
\hline
\end{tabular}

Tabela 2 - Características físicas dos horizontes $A_{1}, A_{3}$ e $B_{22}$ do solo utilizado

Os métodos utilizados para a determinação dos componentes das Tabelas 1 e 2 serão descritos sucintamente, a seguir.

$\mathrm{Ca}^{2+}+\mathrm{Mg}^{2+}$ - extração com solução de $\mathrm{KC} 11 \mathrm{~N}$ e complexação com EDTA 0,025N usando o negro de eriochromo como indicador (VETTORI, 1969).

$\mathrm{Al}^{+3}$ trocável - extração com solução de $\mathrm{KC} 1$ 1N e titulação com solução de $\mathrm{NaOH}$ 0,025N (VETTORI, 1965).

$\mathrm{K}^{+}$e $\mathrm{Na}^{+}$trocáveis - extração com solução $0,025 \mathrm{~N}$ em $\mathrm{H}_{2} \mathrm{SO}_{4}$ e $0,05 \mathrm{~N}$ em $\mathrm{HC} 1$, sendo as leituras feitas em fotômetro de chama (VETTORI, 1969).

$\mathrm{H}^{+}$trocável - extração com solução $1 \mathrm{~N}$ em acetato de cálcio, titulação com solução de $\mathrm{NaOH} 0,1 \mathrm{~N}$ e subtração do $\mathrm{Al}^{3+}$ trocável (VETTORI, 1969). 
C \% - combustão úmida com ácido cromico 0,4N (VETTORI, 1969).

pH - relação solo água 1:2,5 e leitura em potenciômetro (VETTORI, 1969).

Areia, silte e argila - método da pipeta (GROHMANN \& VAN RAIJ, 1974); o tempo de agitação para argila e limo foì calculado pela lei de Stokes (BAVER, 1956).

Superfície específica total - método proposto por HEILMAN et al (1965), que utiliza como fase adsorvida o éter monoetílico do etileno glicol (2-etoxietanol) - EMEG. O cálculo foi efetuado pela fórmula empregada por GROHMANN (1972):

$$
\text { St, } \mathrm{m}^{2} / \mathrm{g}=\frac{\mathrm{mg} \text { EMEG } / \mathrm{g} \text { de terra }}{2,86 \cdot 10^{-4} \mathrm{~g} / \mathrm{m}^{2}}
$$

O método utilizado para a terminação da capacidade de fixação de P foi o descrito por WAUGH \& FITTS (1966). A metodologia empregada será descrita a seguir, em resumo.

Porções de T.F.S.A. equivalentes a $10 \mathrm{~g}$ de T.F.S.E. foram passadas para frascos de Erlenmeyer de $125 \mathrm{ml}$ recebendo, como tratamento, $4 \mathrm{ml}$ de soluções contendo $\mathrm{Ca}\left(\mathrm{H}_{2} \mathrm{PO}_{4}\right)_{2} \cdot \mathrm{H}_{2} \mathrm{O}$ com doses de $\mathrm{P}$ variando de 0 (zero) a $600 \mathrm{ppm}$. No caso da dose 0 (zero), foram juntados $4 \mathrm{ml}$ de água destilada. Os erlenmeyers foram cobertos ficando o conjunto em repouso durante 4 dias, após o que procedeu-se à extração do $\mathrm{P}$ com solução $0,05 \mathrm{~N}$ em $\mathrm{H}_{2} \mathrm{SO}_{4}$. As dosagens foram feitas colorimetricamente segundo CATANI \& JACINTHO (1974), com algumas modificações.

O P fixado foi calculado pela fórmula:

$$
\mathrm{Pf}=\left(\mathrm{Pa}+\mathrm{P}_{\mathrm{s}}\right)-\mathrm{P}_{\mathrm{e}}
$$

$P f=P$ fixado

$\mathrm{Pa}=\mathrm{P}$ adicionado

$\mathrm{P}_{\mathrm{s}}=\mathrm{P}$ existente no solo e extraível com solução $0,05 \mathrm{~N}$ em $\mathrm{H}_{2} \mathrm{SO}_{*}$

$\mathbf{P}_{\mathrm{e}}=\mathbf{P}$ extraído.

\section{RESULTADOS E DISCUSSÃO}

Os resultados obtidos se acham na Tabela 3 . 


\begin{tabular}{|c|c|c|c|c|c|c|}
\hline \multirow{3}{*}{$\begin{array}{c}\mathbf{P} \\
\text { adicionado } \\
\text { ppm }\end{array}$} & \multicolumn{6}{|c|}{$P$ retirado da solução (fixado)* } \\
\hline & \multicolumn{2}{|c|}{$\mathrm{A}_{1}$} & \multicolumn{2}{|c|}{$\mathrm{A}_{3}$} & \multicolumn{2}{|c|}{$\mathrm{B}_{22}$} \\
\hline & $\mathrm{P}, \mathrm{ppm}$ & $\mathrm{P} \%$ & $\mathrm{P}, \mathrm{ppm}$ & $\mathrm{P} \%$ & $\mathrm{P}, \mathrm{ppm}$ & $\mathrm{P} \%$ \\
\hline 0 & - & - & - & - & - & - \\
\hline 50 & 44,47 & 88,94 & 46,89 & 93,78 & 48,25 & 96,50 \\
\hline 100 & 87,83 & 87,83 & 91,49 & 91,49 & 76,76 & 76,76 \\
\hline 150 & 130,65 & 87,09 & 134,09 & 89,39 & 144,12 & 96,08 \\
\hline 200 & 169,89 & 84,94 & 175,42 & 87,11 & 191,66 & 95,83 \\
\hline 250 & 210,88 & 84,35 & 215,89 & 86,36 & 236,43 & 94,57 \\
\hline 300 & 248,47 & 82,82 & 257,40 & 85,80 & 280,52 & 93,50 \\
\hline 400 & 318,34 & 79,57 & 329,77 & 82,44 & 368,44 & 92,11 \\
\hline 500 & 390,41 & 78,54 & 393,62 & 78,72 & 452,73 & 90,54 \\
\hline 600 & 452,38 & 75,39 & 463,01 & 77,17 & 532,32 & 88,72 \\
\hline
\end{tabular}

$\mathrm{CV} \%=1,18$

* Média de três repetições.

Tabela 3 - Fósforo fixado, em $\mathrm{ppm}$ de $\mathrm{P}$ e em porcentagem do $\mathrm{P}$ aplicado.

Observa-se (Tabela 3) que com o aumento da dose de P empregada, a fixação aumentou em quantidades absolutas (ppm de $\mathrm{P}$ fixado) mas diminuiu em quantidades relativas (\% de $\mathrm{P}$ fixado), como já haviam constatado REZENDE (1974), MAGALHÃES (1974) e SOBRAL (1975).

A análise da variância mostrou efeitos altamente significativos para doses de $\mathrm{P}\left(\mathrm{F}=24074,90^{* *}\right)$ e horizontes $\left(\mathrm{F}=1038,53^{* *}\right)$; a interação doses de $\mathrm{P} \times$ horizontes também foi significativa $(\mathrm{F}=77,27 * *)$.

Para efeito de comparação entre as médias foram calculadas as diferenças mínimas significativas pelo teste de Tukey, a 5\%:

Horizontes dentro de doses $-\triangle .=5,60$

Doses dentro de horizontes $-\triangle=7,51$

Na Fig. 1 estão representadas graficamente as relações entre $\mathbf{P}$ extraído e $\mathrm{P}$ adicionado. Pelo processo recomendado por WAUGH \& FITTS (1966) os valores " $X$ " estão aproximadamente em torno de $200 \mathrm{ppm}$ para o horizonte $\mathrm{B}_{22}$ e em torno de $150 \mathrm{ppm}$ para os horizontes $\mathrm{A}_{1} \mathrm{e}_{3}$.

Entretanto, o processo recomendado por WAUGH \& FITTS (1966) não permite uma determinação precisa dos valores " $\mathrm{X}$ ", sobretudo em 
relação aos horizontes $A_{1}$ e $A_{3}$. Por isso, tentou-se também a fórmula proposta por REZENDE (1974).

De acordo com WAUGH \& FITTS (1966) o valor "X" é uma aproximação da quantidade de $\mathrm{P}$ requerida para ultrapassar o efeito da fixação do elemento; a porção restante solúvel, além do valor " $X$ ", permanece disponível às plantas. Para a avaliação de tal valor, REZENDE (1974) usou a seguinte fórmula:

$$
\text { Valor " } \mathrm{X} "=\frac{\mathrm{A}+\mathrm{B}}{2}
$$

sendo A o tratamento que proporcionou uma quantidade de $\mathrm{P}$ extraída, jmediatamente maior que $30 \mathrm{ppm}$ e $\mathrm{B}$ o tratamento que proporcionou uma quantidade de $\mathrm{P}$ extraída imediatamente menor que $30 \mathrm{ppm}$.

O valor $30 \mathrm{ppm}$ foi tomado como nível cítrico baseado no trabalho elaborado por técnicos do PIPAEMG-MG (1972).

Utilizando dados da Tabela 3 , foram então, calculados os valores "X" para os horizontes $A_{1}, A_{3}$ e $B_{22}$, cujos resultados estão na Tabela 4.

\begin{tabular}{lccc}
\hline Horizonte & A (ppm de P) & B (pm de P) & Valor X, (ppm de P) \\
\hline$A_{1}$ & 200 & 150 & 175 \\
$A_{3}$ & 250 & 200 & 225 \\
$B_{22}$ & 400 & 300 & 350 \\
\hline
\end{tabular}

Tabela 4 - Valores “ $\mathrm{X}$ ” dos horizontes $\mathrm{A}_{1}, \mathrm{~A}_{3}$ e $\mathrm{B}_{\varepsilon_{2}}$

A Fig. 1 e os valores " $X$ " encontrados pelo processo de REZENDE (1974) indicam que, dos três horizontes, o $B_{22}$ é o que possui maior poder de retenção de fosfato, seguido pelo horizonte $A_{3}$ e, finalmente, pelo $A_{1}$.

As relações entre as quantidades adicionadas de $P$ (ppm) e as fixadas (ppm) são lineares como mostram as equações de regressão (1), (2) e (3):

Horizonte

$$
\begin{aligned}
& \mathrm{A}_{1} \\
& \mathrm{~A}_{3} \\
& \mathrm{~B}_{22}
\end{aligned}
$$

Equação de regressão

$$
\begin{array}{ll}
\mathrm{y}=17,663+0,744 \mathrm{x} & \mathrm{r}=0,999 * * \\
\mathrm{y}=20,635+0,754 \mathrm{x} & \mathrm{r}=0,998 * * \\
\mathrm{y}=11,654+0,881 \mathrm{x} & \mathrm{r}=0,999
\end{array}
$$


sendo $\mathrm{y}=$ ppm de $\mathrm{P}$ extraída da solução (fixada)

$\mathrm{x}=\mathrm{ppm}$ de $\mathrm{P}$ nas soluções originais.

\section{CONCLUSÃO}

1 - $O$ horizonte $B_{22}$ foi o que apresentou maior capacidade de fixação de fósforo, seguido pelo $\mathrm{A}_{3}$ e, finalmente, pelo $\mathrm{A}_{1}$.

2 - Os valores " $X$ " de WAUGH \& FITTS (1966) encontrados pelo processo apresentado por REZENDE (1974) foram: $350 \mathrm{ppm}, 225 \mathrm{ppm}$ e $175 \mathrm{ppm}$, respectivamente para os horizontes $\mathrm{B}_{22}, \mathrm{~A}_{3}$ e $\mathrm{A}_{1}$.

3 - Houve uma relação muito estreita entre as quantidades de $\mathbf{P}$ adicionadas e as fixadas pelos três horizontes.

SUMMARY

PHOSPHORUS FIXATION BY A LATOSSOL AND DETERMINATION OF THE "X" VALUE OF THE SOIL

An assay was conducted with the aim to evaluate the phosphorus fixation capacity of $\mathrm{A}_{1}(0-22 \mathrm{~cm}), \mathrm{A}_{3}(22-56 \mathrm{~cm})$ and $\mathrm{B}_{22} \quad(155-200 \mathrm{~cm}$ horizons of a "Roxo Latossol". Also, the " $X$ " value of WAUGH \& FITTS (1966) of the three horizons was determinated.

The main results are showed below:

1 - The phosphorus fixation capacity of the three horizons are in the following rank: $\mathrm{B}_{22}>\mathrm{A}_{3}>\mathrm{A}_{1}$.

2 - The " $\mathrm{X}$ " value are: $350 \mathrm{ppm}\left(\mathrm{B}_{22}\right.$ horizon), $225 \mathrm{ppm}\left(\mathrm{A}_{3}\right.$ horizon) and $175 \mathrm{ppm}$ ( $A_{1}$ horizon).

3 - Correlations were found at the level of $1 \%$ between added $\mathrm{P}$ and fixed $\mathrm{P}$ by the studied horizons.

LITERATURA CITADA

BAVER, L.D., 1966 - The mechanical composition of soil. In: Soil Physics, 3.a ed., John Wiley \& Sons, New York, pp. 48-80.

BUCKMAN, H.O. \& N.C. BRADY, 1968 - Natureza e propriedade dos solos. 2. ${ }^{\text {a }}$ ed. Rio de Janeiro, Freitas Bastos, 594 p.

CATANI, R.A. \& A.O. JACINTHO, 1974 - Análise química para avaliar a fertilidade do solo. Bol. Tec. Cient., Esc. Sup. Agric. "Luiz de Queiroz", Piracicaba, n." 37, $54 \mathrm{p}$. 


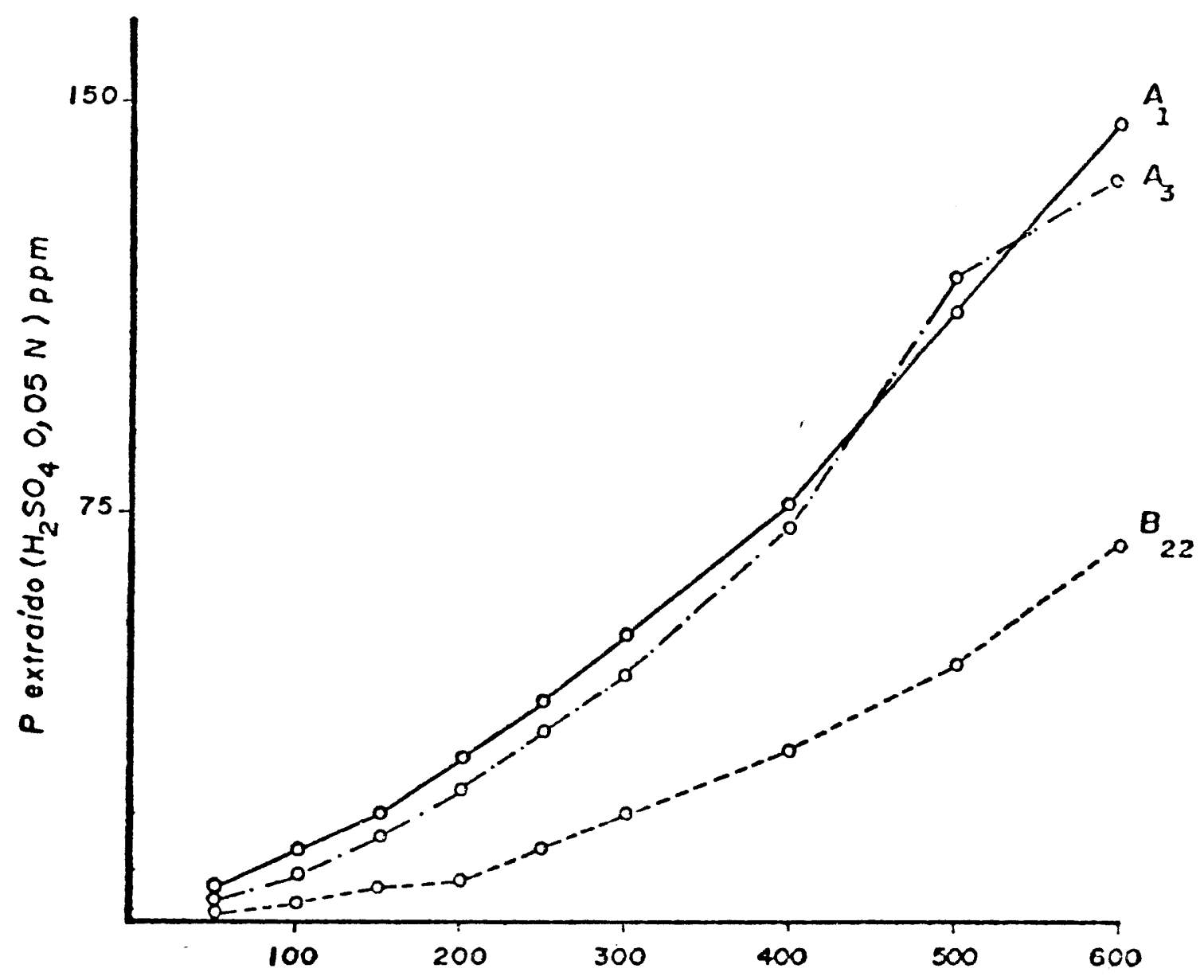

FIGURA 1 - Representação gráfica da relação entre as quantidades de fósforo extraído $\left(\mathrm{H}_{2} \mathrm{SO}_{4} 0,05 \mathrm{~N}\right)$ e adicionado aos horizontes $\mathrm{A}_{1}, \mathrm{~A}_{3}$ e $\mathrm{B}_{22}$ do Latossolo. 
COLE, C.V. \& M.L. JACKSON, 1950 - Solubility equilibrium constant of dihidroxy aluminium dihidrogenphosphate relating to a mechanism of sulphate fexation in soils. Proc. Soil Sci. Soc. Am. Ann Arbor, $15: 84-89$.

DALTON, J.D., C.G. RUSSEL \& D.H. SIELING, 1952 - Effect of organic matter on phosphate availability. Soil Sci., Baltimore, $73: 173-181$.

GROHMANN, F., 1972 - Superfície específica do solo de unidade de mapeamento do Estado de São Paulo. I - Estudo de perfis com horizonte B textural e horizonte B latossólico. Bragantia, $31: 145-165$.

GROHMANN, F. \& B. VAN RAIT, 1974 - Influência dos métodos de agitação na dispersão da argila no solo. Anais do XIV Congr. Bras. de Ciência do Solo, Santa Maria, R.S., $123-132$.

HEILMAN, M.D., K.L. CARTER \& C.L. GONZALEZ, 1965 - The etylene glycol monethyl ether technique for determining soils surface area. Soil Sci. 100:409-413.

HEMWALL, J.B., 1957 - The fixation of phosphorus by soils. Adv. Agron. 9:95-112.

HSU, P.H., 1965 - Fixation of phosphate by aluminum and iron in acidic soils. Soil Sci., New Brunswick, $99: 398-402$.

KARDOS, L.T., 1964 - Soil fixation of plant nutrients. In: BEAR, F.E. Chemistry of the Soil. New York, Reinhold, p. 369-394.

LEAL, J.R. \& A.C.X. VELOSO, 1973 - Adsorção de fosfato em Latossolos sob vegetação de cerrado. Pesq. Agropec. Bras. Ser. Agron., Rio de Janeiro, 8:81-88.

LOURENÇO, S., 1973 - Adsorção e desorção de fósforo em solos do Estado do Paraná. Tese de Doutoramento, ESALQ, Piracicaba, 69 p.

MAGALHÃES, J.C.A.J., 1974 - Efeitos de níveis e modos de aplicação de fósforo na produção de matéria seca e conteúdo deste nutriente no milho (Zea mays, L.), cultivado em solos de "cerrado" de Brasília - D.F. Dissertação de mestrado, ESALQ, Piracicaba, $112 \mathrm{p}$.

PIPAEMG, 1972 - Recomendações do uso de fertilizantes para o Estado de Minas Gerais, 2. ${ }^{a}$ tentativa. Belo Horizonte, 88 p.

REZENDE, T., 1974 - Capacidade de fixação por solos dos Estados da Bahia e Sergipe influência do $\mathrm{pH}$ e tempo de reação. Dissertação de mestrado, ESALQ, Piracicaba, $81 \mathrm{p}$.

SOBRAL, L.F., 1975 - Fixação de fósforo e adubação do feijoeiro (Phaseolus vulgaris, L.) en um Cambisol do Estado de Sergipe. Dissertação de mestrado, ESALQ, Piracicaba, $45 \mathrm{p}$.

STOUT, P.R., 1939 - Alterations in the crystal structures of clay minerals as a result of phosphate fixation. Proc. Soil. Sci. Soc. Am. Ann Arbor, 4:177-182.

VETTORI, L., 1969 - Métodos de análise de solo. Bol. Tec. Equipe Pedol. Fert. Solo, Rio de Janeiro, n. ${ }^{\circ}$, 24 p.

WAUGH, D.L. \& J.W. FITTS, 1966 - Estudos para interpretação de análises de solo: de laboratório e em vasos. Bol. Tec. Int. Soil. Test., Raleigh, n. ${ }^{\circ} 3,33$ p. 
